\title{
Synthesis of flower-like CoOOH with hierarchical micro/nano- structure as a catalyst for peroxymonosulfate activation
}

\author{
Qiang Xia ${ }^{1}$, Lin Zheng ${ }^{1}$, Xiaogang Liao ${ }^{1}$ and Gang $\mathrm{Li}^{{ }^{*}}$ \\ ${ }^{1}$ (School of Chemistry and Chemical Engineering, Chongqing University of Technology, Chongqing 400054, P.R. China)
}

\begin{abstract}
In this work, hierarchical flower-like $\mathrm{CoOOH}$ assembled from many nanoflakes with a thickness of $20 \mathrm{~nm}$ was fabricated by a simple chemical bath deposition (CBD) method, and its performance as a heterogenous catalyst to activate peroxymonosulfate (PMS) for degradation of methylene blue (MB) solution was explored. The results show that $\mathrm{CoOOH} / \mathrm{PMS}$ is a high efficient system for MB removal, in which $91.95 \%$ degradation of MB can be realized within $25 \mathrm{~min}$. Influences of process parameters, including dosage of PMS, addition of catalyst, and co-existed anions on $\mathrm{MB}$ degradation were also investigated. It is found that the degradation rate of MB increases with the increase of the amount of PMS from $0.15 \mathrm{mmol} \cdot \mathrm{L}^{-1}$ to 0.45 $\mathrm{mmol} \cdot \mathrm{L}^{-1}$. In such PMS concentrations ranges, CoOOH catalyst amount of $0.03 \mathrm{~g} \cdot \mathrm{L}^{-1}$ is adequate for PMS activation in the reaction system. The presence of $\mathrm{Cl}^{-}$and $\mathrm{HCO}_{3}{ }^{-}$anions has a negative effect on $\mathrm{MB}$ degradation, while $\mathrm{SO}_{4}{ }^{2-}$ has no significant influence. In addition, quenching experiments were conducted and $\mathrm{SO}_{4}{ }^{--}$is confirmed as the main active specie during the MB degradation process. Moreover, cycling runs proven that $\mathrm{CoOOH}$ could be re-used to activate PMS for MB degradation without notable activity reduction. These results suggest that hierarchical $\mathrm{CoOOH}$ is a promising PMS activator for organic pollutant removal.
\end{abstract}

\section{Introduction}

With the rapid development of economy, water pollution is becoming more and more serious. Among the industrial effluents, dyeing wastewater discharged from textile, printing and other industries are considered extremely detrimental to ecological environment and human health due to their deep chromaticity, high organic content, poor biodegradability and potential carcinogenicity. Advanced oxidation processes (AOPs), including photocatalysis, fenton reaction and catalytic ozonation, were regarded as promising techniques to deal with dye wastewater. It is usually believed that the excellent mineralization efficiency towards organic components by AOPs is attributed to the formation of hydroxyl radical $(\cdot \mathrm{OH})$, which possesses high redox potential $(\mathrm{E} \theta=2.81 \mathrm{~V})$ and non-selective strong oxidation ability. Nevertheless, the above-mentioned approaches generally suffer from many limitations, such as ultraviolet light requirement, sludge generation and essential special equipment, which therefore greatly influences their practical applications.

Recently, AOPs based on sulfate radical (SO4•-) has attracted more and more peoples' attention. This is principally because $\mathrm{SO} 4 \cdot-$ has more advantages in organic compounds degradation process when compared with $\cdot \mathrm{OH}$, such as higher redox potential $(\mathrm{E} \theta=2.5 \sim 3.1 \mathrm{~V})$, longer half-life period (approximately 30 40 $\mu$ s), and wider $\mathrm{pH}$ adaptation range [1-2]. Therefore, $\mathrm{SO} 4 \cdot$ - based AOPs show a great potential in organic waste-water treatment. In general, SO4•- can be produced by the activation of peroxymonosulfate (PMS) or persulfate (PS) [3]. Those activation methods consist of heat, ultraviolet light, ultrasound and catalysts [1]. Among all these strategies, catalytic activation may be the best choice due to its convenience, high efficiency and no need for continuous energy replenishment. It has been reported that $\mathrm{Co} 2+$ is the most efficient homogeneous catalyst for PMS activation [4]. However, it is very difficult to recovery the heavy metal ion from the water body after activation, which will inevitably cause a secondary pollution to water environment. Instead, heterogeneous cobalt-based catalysts can provide a much better option for PMS activation due to their easiness to recycle, although they always show slightly inferior performance than that of homogeneous counterparts. Up to now, various cobalt-based heterogeneous catalysts, such as cobalt oxides, cobalt-doped catalysts, and bimetallic oxide materials [4-8], have been developed for PMS activation. It is found that the performances of these catalysts are highly associated with their chemical composition, morphology, preparation method and microstructure. Thus, from a practical point of view, fabrication of new-type cobalt-based materials with new components, novel shapes and micro-structures and further exploration of their properties towards PMS activation is highly desirable. $\mathrm{CoOOH}$, a member of the cobalt-based materials, is commonly used as electrode materials or precursors for preparation of $\mathrm{Co} 3 \mathrm{O} 4$. Unfortunately, to the best of our knowledge, no research is reported on the

\footnotetext{
* Corresponding author. Tel.: +86 23 62563221. E-mail address: 47922138@163.com (G. Li)
} 
$\mathrm{CoOOH}$ as a catalyst for PMS activation. In this study, $\mathrm{CoOOH}$ with hierarchical micro-nano structure was synthesized by a simple chemical bath deposition (CBD) method and its catalytic ability in PMS activation for MB degradation was systematically investigated. Meanwhile, the mechanism of PMS activation by $\mathrm{CoOOH}$ was also studied.

\section{Materials and methods}

All of the reagents were of analytical grade and were used without further purification. All solutions were prepared with deionized water. $\mathrm{CoOOH}$ was prepared through a simple CBD method. Typically, $\mathrm{CoSO}_{4} \cdot 7 \mathrm{H}_{2} \mathrm{O}$ and $\mathrm{K}_{2} \mathrm{~S}_{2} \mathrm{O}_{8}$ were dissolved in $400 \mathrm{~mL}$ deionized water in sequence to achieve a concentration of $0.25 \mathrm{~mol} \cdot \mathrm{L}^{-1}$ and $0.0625 \mathrm{~mol} \cdot \mathrm{L}^{-1}$, respectively. Then, under intense stirring, $15 \mathrm{~mL}$ concentrated ammonia (wt. $25 \%-28 \%$ ) was rapidly poured into the aforementioned solution. After that, the reaction mixture was continuously stirred for another 4 hours. Next, the precipitation was collected by filtration, washed with deionized water and absolute ethanol for several times. After drying at $60{ }^{\circ} \mathrm{C}$ for 5 hours, the final tawny catalysts were then obtained.

Powder X-ray diffraction (XRD) patterns were recorded by a Shimadzu XRD-7000 X-ray diffractometer with $\mathrm{Cu} \mathrm{K \alpha}$ radiation $(40 \mathrm{kV}, 30 \mathrm{~mA})$, over a $2 \theta$ range from $10^{\circ}$ to $80^{\circ}$ at room temperature. Morphology of the sample was observed on a Quanta 600F scanning electron microscope (SEM).

Methylene blue (MB) solution was selected as simulated organic wastewater to evaluate the catalytic activity of $\mathrm{CoOOH}$ for activating PMS. The catalytic experiments were performed in a $1000 \mathrm{~mL}$ beaker at $25^{\circ} \mathrm{C}$. Before the addition of PMS, a certain amount of catalysts was dispersed in $500 \mathrm{~mL} \mathrm{MB}$ solution with an initial concentration of $10 \mathrm{mg} \cdot \mathrm{L}^{-1}$ under constant magnetic stirred for $50 \mathrm{~min}$ to achieve the adsorption-desorption balance (the adsorption rate was measured as $3.43 \%$ ). After the reaction was initiated by PMS, $3.5 \mathrm{~mL}$ of the reaction mixture was withdrawn at a given time interval, which was then filtered through a $0.22 \mu \mathrm{m}$ nylon membrane filter and immediately mixed with $\mathrm{NaNO}_{2}(3$ $\left.\mathrm{mmol} \cdot \mathrm{L}^{-1}, 150 \mu \mathrm{L}\right)$ to quench the reaction.

\section{Results and discussion}

\subsection{Characterization results}

Figure 1(a) displays the XRD pattern of the obtained sample. Three characteristic diffraction peaks located at $2 \theta=22.5^{\circ}, 35.3^{\circ}$ and $61.2^{\circ}$ are observed, which can be well indexed to the ( $\left.\begin{array}{lll}1 & 1 & 0\end{array}\right),\left(\begin{array}{lll}1 & 3 & 0\end{array}\right)$ and $\left(\begin{array}{lll}2 & 3 & 1\end{array}\right)$ crystalline planes of CoOOH (JCPDS\# 26-0480), respectively. The wide diffraction peaks indicate the low crystallinity of $\mathrm{CoOOH}$ synthesized by the CBD method. Figure 1(b-c) displays the SEM images of the sample with different magnification. As shown in figure 1(b), powder $\mathrm{CoOOH}$ is composed of numerous uniform flower-like particles with a diameter of $350 \mathrm{~nm}$. From the magnified FESEM image (figure 1c), it is clear that these flower-like particles are hierarchically micro-nano structured, all of which are assembled from nanosheets with a thickness of $20 \mathrm{~nm}$.
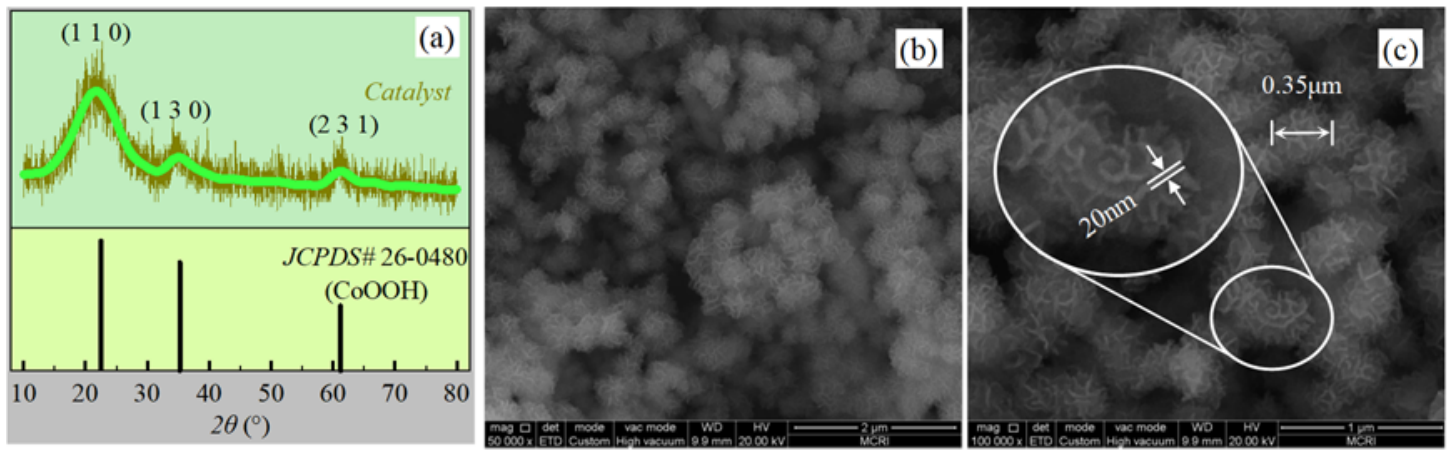

Fig 1. XRD pattern (a) and SEM images (b-c) of the sample.

\subsection{Degradation of MB by $\mathrm{CoOOH}$-activated PMS}

The removal efficiency of MB by $\mathrm{CoOOH} / \mathrm{PMS}$ system was presented in figure 2 . As shown in figure $2(\mathrm{a})$, the removal rate of $\mathrm{MB}$ was only $14.55 \%$ after a reaction time of $25 \mathrm{~min}$, when PMS was alone added to the solution. However, a degradation rate of $91.95 \%$ can be reached in the presence of both PMS and $\mathrm{CoOOH}$. This suggests that $\mathrm{CoOOH}$ was highly active towards PMS activation to generate radicals to decolorize MB solution. Influences of PMS concentration and catalyst dosage on MB degradation were then investigated. It is found that PMS concentration plays an important role in $\mathrm{MB}$ degradation. At a fixed addition amount of $\mathrm{CoOOH}\left(0.03 \mathrm{~g} \cdot \mathrm{L}^{-1}\right)$, the removal efficiencies of MB with PMS concentrations of $0.15 \mathrm{mmol} \cdot \mathrm{L}^{-1}, 0.25 \mathrm{mmol} \cdot \mathrm{L}^{-1}, 0.35 \mathrm{mmol} \cdot \mathrm{L}^{-1}$ and 0.45 $\mathrm{mmol} \cdot \mathrm{L}^{-1}$ are $53.06 \%, 72.64 \%, 91.95 \%$, and $97.05 \%$, respectively. 

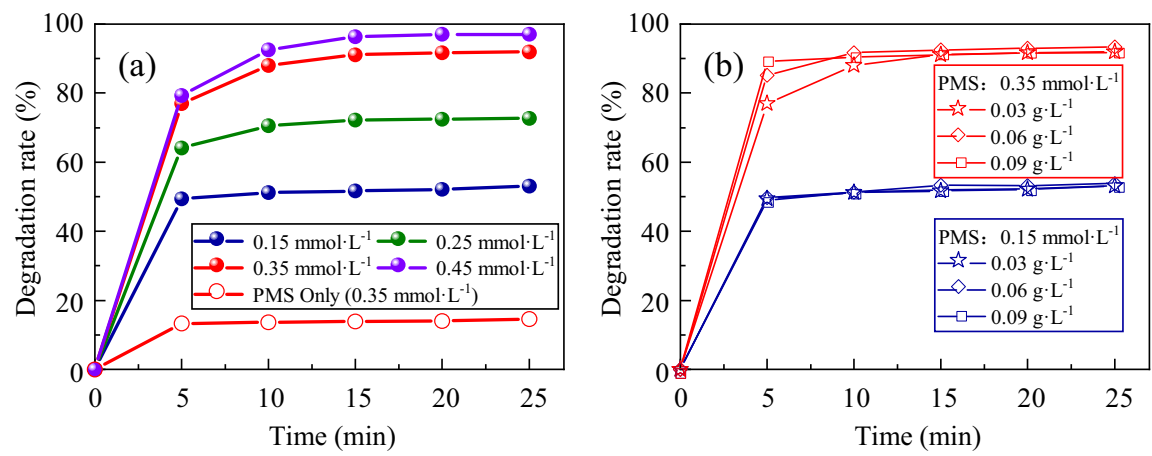

Fig 2. Effects of PMS concentration (a) and catalyst dosage (b) on the degradation of MB by PMS/CoOOH system. Reaction conditions: $\mathrm{T}=25^{\circ} \mathrm{C}, \mathrm{V}_{(\mathrm{MB})}=500 \mathrm{~mL}, \mathrm{C}_{(\mathrm{MB})}=10 \mathrm{mg} \cdot \mathrm{L}^{-1}$.

Namely, with the increase of PMS concentration in the solution, much higher $\mathrm{MB}$ degradation rate can be achieved. This is due to the fact that more free radicals can be produced when higher concentration of PMS was introduced. By contrast, catalyst dosage has little effect on MB degradation process as depicted in figure 2(b). This signifies that the catalytic active sites provided by 0.03 $\mathrm{g} \cdot \mathrm{L}^{-1} \mathrm{CoOOH}$ was adequate for PMS activation when PMS concentration ranges from 0.15 to $0.35 \mathrm{mmol} \cdot \mathrm{L}^{-1}$ in the reaction system.

\subsection{Effects of coexisted inorganic anions}

Considering the composition complexity of the practical wastewater, effect of coexisted anions on degradation of $\mathrm{MB}$ by $\mathrm{CoOOH} / \mathrm{PMS}$ system was further studied. Three kinds of common and typical inorganic anions, $\mathrm{Cl}^{-}, \mathrm{SO}_{4}{ }^{2-}$, and $\mathrm{HCO}_{3}{ }^{-}$were chosen as the model. They were introduced into the MB solution in the form of sodium salt and their concentrations were both kept as $20 \mathrm{mmol} \cdot \mathrm{L}^{-1}$. As shown in figure 3 , introduction of $\mathrm{SO}_{4}^{2-}$ has no effect on the MB degradation. However, obvious inhibition of MB degradation can be observed after the introduction of $\mathrm{Cl}^{-}$and $\mathrm{HCO}_{3}{ }^{-}$, whose degradation rate decreased from $91.95 \%$ to $56.60 \%$ and $23.48 \%$, respectively.

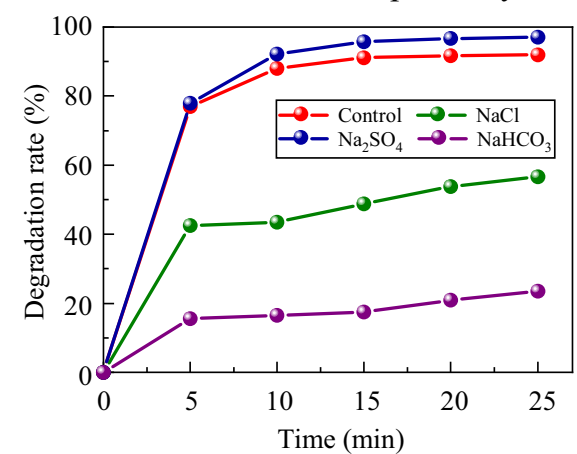

Fig 3. Effect of different coexisted inorganic anions on MB degradation. Reaction conditions: $\mathrm{T}=25^{\circ} \mathrm{C}, \mathrm{V}_{(\mathrm{MB})}$ $=500 \mathrm{~mL}, \mathrm{C}_{\text {(anions) }}=20 \mathrm{mmol} \cdot \mathrm{L}^{-1}, \mathrm{C}_{0(\mathrm{MB})}=10 \mathrm{mg} \cdot \mathrm{L}^{-1}$, $\mathrm{C}_{0(\mathrm{PMS})}=0.35 \mathrm{mmol} \cdot \mathrm{L}^{-1}$, and catalyst dosage $=0.03$ $\mathrm{g} \cdot \mathrm{L}^{-1}$.
The inhibition effect may be correlated with the consumption of $\mathrm{SO}_{4}{ }^{-}$in the presence of $\mathrm{Cl}^{-}$and $\mathrm{HCO}_{3}{ }^{-}$, which can be explained with the following reaction equations [9-10].

$$
\begin{gathered}
\mathrm{Cl}^{-}+\mathrm{SO}_{4}^{--} \rightarrow \mathrm{Cl}^{\bullet}+\mathrm{SO}_{4}^{2-} \\
\mathrm{HCO}_{3}^{-}+\mathrm{SO}_{4}^{--} \rightarrow \mathrm{HCO}_{3}^{\bullet}+\mathrm{SO}_{4}^{2-}
\end{gathered}
$$

As illustrated, new radicals of $\mathrm{Cl}^{\circ}$ and $\mathrm{HCO}_{3}{ }^{\circ}$ were formed by reaction of $\mathrm{SO}_{4}{ }^{-}$and the corresponding anions, but their oxidation capacities were not as effective as that of $\mathrm{SO}_{4}{ }^{\circ}$, which thus resulted in a worse $\mathrm{MB}$ degradation efficiency.

\subsection{Activation mechanism of PMS by $\mathrm{CoOOH}$}

As is well known, active intermediates generated from PMS activated by catalyst was responsible for the high reactivity towards organic compounds removal $[1,2,4]$. And it is generally accepted that $\mathrm{SO}_{4}{ }^{-}$and $\cdot \mathrm{OH}$ were the main reactive oxygen species[11]. To explore the particular activation mechanism of PMS catalyzed by $\mathrm{CoOOH}, \mathrm{Et}-\mathrm{OH}$ and TBA, which were commonly used as scavengers for $\mathrm{SO}_{4} \cdot-\& \cdot \mathrm{OH}$ or $\cdot \mathrm{OH}$, respectively $[1,10]$, were introduced into the reaction system. It should be noted that the total volume of $\mathrm{MB}$ solution remained unchanged when Et-OH or TBA were added. Figure 4 exhibited the results of radical capture experiments. It is clear that degradation rate of $\mathrm{MB}$ decreased as the scavenger concentration rose for both Et-OH and TBA. However, Et-OH showed a much higher inhibition effect than that of TBA for removal of MB at the same scavenger concentration. This indicates that $\cdot \mathrm{OH}$ and $\mathrm{SO}_{4}{ }^{-}$were both generated from PMS activation with $\mathrm{CoOOH}$, but $\mathrm{SO}_{4}{ }^{--}$was the primary reactive specie during the degradation process of MB. 

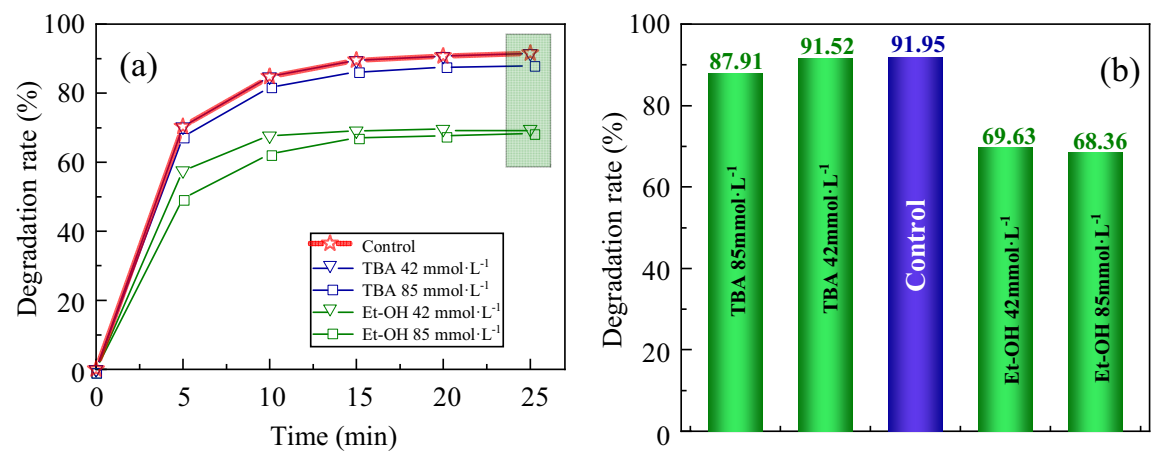

Fig 4. Effect of radical scavengers on $\mathrm{MB}$ degradation (a-b) within 25 min reaction. Reaction conditions: $\mathrm{T}=25{ }^{\circ} \mathrm{C}$, $\mathrm{V}_{(\mathrm{MB})}=500 \mathrm{~mL}, \mathrm{C}_{0(\mathrm{MB})}=10 \mathrm{mg} \cdot \mathrm{L}^{-1}, \mathrm{C}_{0(\mathrm{PMS})}=0.35 \mathrm{mmol} \cdot \mathrm{L}^{-1}$, and catalyst dosage $\left.=0.03 \mathrm{~g} \cdot \mathrm{L}^{-1}\right)$.

Based on previous literature reported[4] and our experimental results, a possible reaction mechanism of MB degradation in the system of $\mathrm{CoOOH} / \mathrm{PMS}$ was proposed as follows.

$$
\begin{aligned}
& \equiv \mathrm{Co}(\mathrm{III})+\mathrm{HSO}_{5}^{-} \rightarrow \equiv \mathrm{Co}(\mathrm{II})+\mathrm{SO}_{5}^{--}+\mathrm{H}^{+} \\
& \equiv \mathrm{Co}(\mathrm{II})+\mathrm{HSO}_{5}^{-} \rightarrow \equiv \mathrm{Co}(\mathrm{III})+\mathrm{SO}_{4}^{--}+\mathrm{OH}^{-} \\
& \mathrm{HSO}_{5}^{-} \stackrel{=\mathrm{CoOOH}^{-}}{\longrightarrow} \mathrm{SO}_{4}^{--}+{ }^{-} \mathrm{OH} \\
& \mathrm{MB}+\mathrm{SO}_{4}^{--} / \cdot \mathrm{OH} \rightarrow \text { Intermediates } \rightarrow \mathrm{H}_{2} \mathrm{O}+\mathrm{CO}_{2}
\end{aligned}
$$

\subsection{Reusability of the catalyst}

Cycle experiments were carried out for estimating the stability and re-usability of the hierarchical flower-like $\mathrm{CoOOH}$ catalyst. As seen in figure 5, in the four consecutive cycles, MB degradation rates were $91.95 \%$, $88.27 \%, 83.35 \%$ and $84.26 \%$, respectively, which showed a slight downward trend.

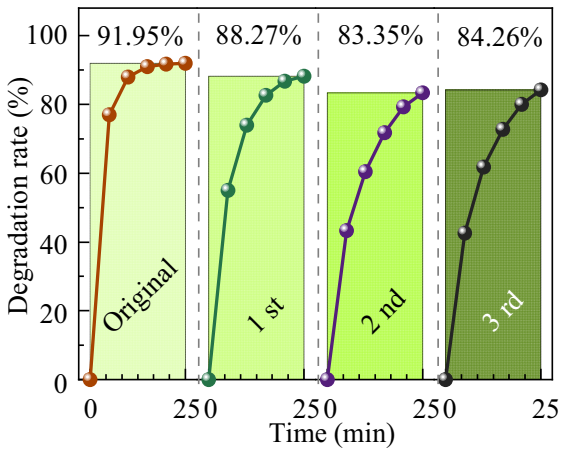

Fig 5. Cycling runs for the removal of $\mathrm{MB}$ in the $\mathrm{CoOOH} / \mathrm{PMS}$ system. Reaction conditions: $\mathrm{T}=25^{\circ} \mathrm{C}$, $\mathrm{V}_{(\mathrm{MB})}=500 \mathrm{~mL}, \mathrm{C}_{0(\mathrm{MB})}=10 \mathrm{mg} \cdot \mathrm{L}^{-1}, \mathrm{C}_{0(\mathrm{PMS})}=0.35$ $\mathrm{mmol} \cdot \mathrm{L}^{-1}$, and catalyst dosage $=0.03 \mathrm{~g} \cdot \mathrm{L}^{-1}$.

This may be due to the loss of a small amount of active components in the catalyst during cycling runs. Fortunately, degradation rate of MB kept steady from the second run. Overall, the flower-like hierarchical $\mathrm{CoOOH}$ obtained by the CBD method shows a good stability and re-usability.

\section{Conclusions}

In this study, hierarchical micro/nano structured $\mathrm{CoOOH}$ with flower-like morphology was synthesized by the CBD method, and it was used as a heterogeneous catalyst for
PMS activation to degrade MB solution. The effects of key parameters (PMS concentration, catalyst dosage and co-existed anions in solution) on MB degradation were investigated in detail. Results indicated that the degradation rate of $\mathrm{MB}$ increased gradually with the increase of the PMS concentration ranging from 0.15 $\mathrm{mmol} \cdot \mathrm{L}^{-1}$ to $0.45 \mathrm{mmol} \cdot \mathrm{L}^{-1}$. Under such PMS concentration ranges, $\mathrm{CoOOH}$ with an addition amount of $0.03 \mathrm{~g} \cdot \mathrm{L}^{-1}$ was enough for PMS activation in the reaction system. The presence of $\mathrm{Cl}^{-}$and $\mathrm{HCO}_{3}{ }^{-}$anions had a negative effect on $\mathrm{MB}$ degradation, while $\mathrm{SO}_{4}{ }^{2-}$ had no significant influence. Under the optimum reaction conditions, $91.95 \% \mathrm{MB}$ could be degraded within $25 \mathrm{~min}$ in $\mathrm{CoOOH} / \mathrm{PMS}$ system. In addition, quenching tests confirmed that $\mathrm{SO}_{4}{ }^{--}$was the main active specie during the MB degradation process. Moreover, owing to its hierarchical structure, $\mathrm{CoOOH}$ showed a good stability and re-usability. After four consecutive cycles, MB removal rate was still reached up to $84.26 \%$. These results indicate that hierarchical $\mathrm{CoOOH}$ is a promising PMS activator for organic pollutant removal.

\section{Acknowledgements}

This work was supported by Research Program of Chongqing university of technology (KLA20031 and clgycx 20203085).

\section{References}

1. Ghanbari, F., Moradi, M. (2017) Application of peroxymonosulfate and its activation methods for degradation of environmental organic pollutants: review. Chemical Engineering Journal, 310: 41-62.

2. Wang, J.L., Wang, S.Z. (2018) Activation of persulfate (PS) and peroxymonosulfate (PMS) and application for the degradation of emerging contaminants. Chemical Engineering Journal, 334: 1502-1517.

3. Zhou, Y., Jiang, J., Gao, Y., Ma, J., Pang, S.Y., Li, J., Lu, X.T., Yuan, L.P. (2015) Activation of peroxymonosulfate by benzoquinone: A novel nonradical oxidation process. Environmental Science \& Technology, 49: 12941-12950. 
4. Hu, P.D., Long M.C. (2016) Cobalt-catalyzed sulfate radical-based advanced oxidation: a review on heterogeneous catalysts and applications. Applied Catalysis B: Environmental, 181: 103-117.

5. Oh W.D., Chang, V.W.C., Hu, Z.T., Goei, R., Lim, T.T. (2017) Enhancing the catalytic activity of g$\mathrm{C} 3 \mathrm{~N} 4$ through $\mathrm{Me}$ doping $(\mathrm{Me}=\mathrm{Cu}, \mathrm{Co}$ and $\mathrm{Fe})$ for selective sulfathiazole degradation via redox-based advanced oxidation process. Chemical Engineering Journal, 323: 260-269.

6. Qin, Q.D., Gao, X., Wu, X., Liu, Y.H. (2019) $\mathrm{NaBH} 4-$ treated cobalt-doped g-C3N4 for enhanced activation of peroxymonosulfate. Materials Letters, 256: 126623-126626.

7. Qin, F.X., Jia, S.Y., Liu, Y., Han, X., Ren, H.T., Zhang, W.W., Hou, J.W., Wu, S.H. (2013) Metalorganic framework as a template for synthesis of magnetic $\mathrm{CoFe} 2 \mathrm{O} 4$ nanocomposites for phenol degradation. Materials Letters, 101: 93-95.

8. Tian, X.K., Tian, C., Nie, Y.L., Dai, C., Yang, C., Tian N., Zhou, Z.X., Li, Y.,Wang, Y.X. (2018) Controlled synthesis of dandelion-like NiCo2O4 microspheres and their catalytic performance for peroxymonosulfate activation in humic acid degradation. Chemical Engineering Journal, 331: 144-151.

9. Sun, Z.M., Liu, X.R., Dong, X.B., Zhang, X.W., Tan, Y., Yuan, F., Zheng, S.L., Li, C.Q. (2021) Synergistic activation of peroxymonosulfate via in situ growth FeCo2O4 nanoparticles on natural rectorite: role of transition metal ions and hydroxyl groups. Chemosphere, 263: 127965.

10. Shad, A., Chen, J., Qu, R.J., Ahmed Dar, A., BinJumah, M., Allam, A.A., Wang, Z.Y. (2020) Degradation of sulfadimethoxine in phosphate buffer solution by UV alone, UV/PMS and UV/H2O2: kinetics, degradation products, and reaction pathways. Chemical Engineering Journal, 398: 125357.

11. Khan, S., He, X., Khan, H.M., Boccelli, D., Dionysiou, D.D. (2016) Efficient degradation of lindane in aqueous solution by iron(II) and/or UV activated peroxymonosulfate. Journal of Photochemistry and Photobiology A: Chemistry, 316 37-43. 